\title{
A Class of Integral Operators Preserving Subordination and Superordination for Analytic Functions
}

\author{
H. A. Al-Kharsani, ${ }^{1,2}$ N. M. Al-Areefi, ${ }^{1,2}$ and Janusz Sokó1 ${ }^{1,2}$ \\ ${ }^{1}$ Department of Mathematics, Faculty of Science, P.O. Box 838, Dammam 31113, Saudi Arabia \\ ${ }^{2}$ Department of Mathematics, Rzeszów University of Technology, Ul. W. Pola 2, 35-959 Rzeszów, Poland
}

Correspondence should be addressed to N. M. Al-Areefi, najarifi@hotmail.com

Received 8 May 2012; Accepted 26 June 2012

Academic Editors: J. Cui, J.-L. Wu, and C. Zhu

Copyright (c) 2012 H. A. Al-Kharsani et al. This is an open access article distributed under the Creative Commons Attribution License, which permits unrestricted use, distribution, and reproduction in any medium, provided the original work is properly cited.

The purpose of the paper is to investigate several subordination- and superordination-preserving properties of a class of integral operators, which are defined on the space of analytic functions in the open unit disk. The sandwich-type theorem for these integral operators is also presented. Moreover, we consider an application of the subordination and superordination theorem to the Gauss hypergeometric function.

\section{Introduction}

Let $\mathscr{t}$ be the class of functions analytic in the unit disk $U=\{z \in \mathbb{C}:|z|<1\}$, and denote by $A$ the class of analytic functions in $U$ and usually normalized, that is, $A=\{f \in \mathscr{d}$ : $\left.f(0)=1, f^{\prime}(0)=1\right\}$. The function $f \in \mathscr{d}$ is said to be subordinate to $F \in \mathscr{d}$, or $F$ is said to be superordinate to $f$, if there exists a function $w \in \mathscr{d}$ such that

$$
\begin{gathered}
w(0)=0, \quad|w(z)|<1 \quad(z \in \mathbb{U}), \\
f(z)=F(w(z)) \quad(z \in \mathbb{U}) .
\end{gathered}
$$

In this case, we write

$$
f \prec F \quad(z \in \mathbb{U}) \quad \text { or } \quad f(z) \prec F(z) \quad(z \in \mathbb{U}) .
$$


If the function $F$ is univalent in $\mathbb{U}$, then we have (cf. [1])

$$
f \prec F \quad(z \in \mathbb{U}) \Longleftrightarrow f(0)=F(0), \quad f(\mathbb{U}) \subset F(\mathbb{U})
$$

Definition 1.1 (Miller and Mocanu [1]). Let

$$
\phi: \mathbb{C}^{2} \longrightarrow \mathbb{C}
$$

and let $h$ be univalent in $\mathbb{U}$. If $p$ is analytic in $\mathbb{U}$ and satisfies the following differential subordination:

$$
\phi\left(p(z), z p^{\prime}(z)\right)<h(z) \quad(z \in \mathbb{U}),
$$

then $p$ is called a solution of the differential subordination. A univalent function $q$ is called a dominant of the solutions of the differential subordination or, more simply, a dominant if $p \prec q$ for all $p$ satisfying the differential subordination (1.5). A dominant $\tilde{q}$ that satisfies $\tilde{q} \prec q$ for all dominants $q$ of (1.5) is said to be the best dominant.

Definition 1.2 (Miller and Mocanu [2]). Let $\varphi: \mathbb{C}^{2} \rightarrow \mathbb{C}$ and let $h$ be analytic in $\mathbb{U}$. If $p$ and $\varphi\left(p(z), z p^{\prime}(z)\right)$ are univalent in $\mathbb{U}$ and satisfy the following differential superordination:

$$
h(z) \prec \varphi\left(p(z), z p^{\prime}(z)\right) \quad(z \in \mathbb{U}),
$$

then $p$ is called a solution of the differential superordination. An analytic function $q$ is called a subordinat of the solutions of the differential superordination or, more simply, a subordinant if $q \prec p$ for all $p$ satisfying the differential superordination (1.6). A univalent subordinat $\tilde{q}$ that satisfies $q<\tilde{q}$ for all subordinats $q$ of (1.6) is said to be the best subordinat.

Definition 1.3 (Miller and Mocanu [2]). We denote by $Q$ the class of functions $f$ that are analytic and injective on $\overline{\mathbb{U}} \backslash E(f)$, where

$$
E(f):\left\{\zeta: \zeta \in \partial \mathbb{U}, \lim _{z \rightarrow \zeta} f(z)=\infty\right\}
$$

and are such that

$$
f^{\prime}(\zeta) \neq 0, \quad \zeta \in \partial \mathbb{U} \backslash E(f)
$$

We define the family of integral operators $I_{\beta, \gamma}^{h} f(z)$ as follows:

$$
I_{\beta, \gamma}^{h} f(z)=\left[\frac{\gamma+\beta}{z^{\gamma}} \int_{0}^{z} f^{\beta}(t) h^{\gamma-1}(t) h^{\prime}(t) d t\right]^{1 / \beta},
$$

where each of the functions $f$ and $h$ belong to the class $A$ and the parmeters $\beta \in C \backslash\{0\}, \gamma \in C$, $\operatorname{Re}(\gamma+\beta)>0$, were so constrained that the integral operators in (1.9) exist. 
Throughout this paper, we will denote by $\mathcal{A}_{\beta, \gamma}$ the following analytic function class:

$$
\mathcal{A}_{\beta, \gamma}=\left\{f \in \mathscr{\ell}(\mathbb{U}): \frac{f(z)}{z} \neq 0, \frac{I_{\beta, \gamma}(z)}{z} \neq 0,(z \in U)\right\}
$$

This integral operator $I_{\beta, \gamma}^{h}(f)$ defined by (1.9) has been extensively studied by authors [3-6] with suitable restriction on the parameters $\beta$ and $\gamma$.

In particular, if we take $\gamma=0$ we get the integral operator defined by Bulboacă [7-12] and if we put $h(t)=t$ in (1.9), we will get the results in [13].

In the present paper, we obtain the subordination- and superordination-preserving properties of the integral operator $I_{\beta, \gamma}^{h}(f)$ defined by (1.9) with the sandwich-type theorem. We also consider an interesting application of our main results to the Gauss hypergeometric function.

The following lemmas will be required in our present investigation.

Lemma 1.4 (Miller and Mocanu [14]). Suppose that the function

$$
H: \mathbb{C}^{2} \longrightarrow \mathbb{C}
$$

satisfies the following condition:

$$
\mathfrak{R e}\{H(\text { is }, t)\} \leqq 0
$$

for all real s and for all

$$
t \leq-\frac{1}{2} n\left(1+s^{2}\right) \quad(n \in \mathbb{N}:=\{1,2,3, \ldots\}) .
$$

If the function

$$
p(z)=1+p_{n} z^{n}+\cdots
$$

is analytic in $\mathbb{U}$ and

$$
\mathfrak{R e}\left\{H\left(p(z), z p^{\prime}(z)\right)\right\}>0 \quad(z \in \mathbb{U}),
$$

then

$$
\mathfrak{R e}\{p(z)\}>0 \quad(z \in \mathbb{U}) .
$$

Lemma 1.5 (Miller and Mocanu [15]). Let $\beta, \gamma \in \mathbb{C}$ with $\beta \neq 0$ and let $h \in \mathcal{t l}$ with $h(0)=c$. If

$$
\mathfrak{R e}\{\beta h(z)+\gamma\}>0 \quad(z \in \mathbb{U}),
$$


then the solution $q, q(0)=c$, of the following differential equation:

$$
q(z)+\frac{z q^{\prime}(z)}{\beta q(z)+\gamma}=h(z)
$$

is analytic in $\mathbb{U}$ and satisfies the inequality given by

$$
\mathfrak{R e}\{\beta q(z)+\gamma\}>0 \quad(z \in \mathbb{U}) .
$$

Lemma 1.6 (Miller and Mocanu [1]). Let $p \in \mathcal{Q}$ with $p(0)=a$ and let

$$
q(z)=a+a_{n} z^{n}+\cdots
$$

be analytic in $\mathbb{U}$ with

$$
q(z) \not \equiv a, \quad n \in \mathbb{N} .
$$

If $q$ is not subordinate to $p$, then there exist points

$$
z_{0}=r_{0} e^{i \theta} \in \mathbb{U}, \quad \zeta_{0} \in \partial \mathbb{U} \backslash E(f),
$$

for which

$$
q\left(\mathbb{U}_{r_{0}}\right) \subset p(\mathbb{U}), \quad q\left(z_{0}\right)=p\left(\zeta_{0}\right), \quad z_{0} q^{\prime}\left(z_{0}\right)=m \zeta_{0} p^{\prime}\left(\zeta_{0}\right) \quad(m \geqq n) .
$$

Our next lemmas deal with the notion of subordination chain. A function $L(z, t)$ defined on $\mathbb{U} \times[0, \infty)$ is called the subordination chain (or Löwner chain) if $L(z, t)$ is analytic and univalent in $\mathbb{U}$ for all $t \in[0, \infty), L(z, t)$ is continuously differentiable on $[0, \infty)$ for all $z \in \mathbb{U}$ and

$$
L(z, s)<L(z, t) \quad(z \in \mathbb{U} ; 0 \leqq s<t) .
$$

Lemma 1.7 (Miller and Mocanu [2]). Let $\mathscr{L}[a, 1]=\left\{f \in \mathscr{L}: f(0)=a, f^{\prime}(0) \neq 0\right\}$ and

$$
q \in \mathscr{H}[a, 1], \quad \varphi: \mathbb{C}^{2} \rightarrow \mathbb{C} .
$$

Also set

$$
\varphi\left(q(z), z q^{\prime}(z)\right) \equiv h(z) \quad(z \in \mathbb{U}) .
$$

If

$$
L(z, t):=\varphi\left(q(z), t z q^{\prime}(z)\right)
$$


is a subordination chain and

$$
p \in \mathscr{H}[a, 1] \cap Q
$$

then

$$
h(z) \prec \varphi\left(p(z), z p^{\prime}(z)\right) \quad(z \in \mathbb{U})
$$

implies that

$$
q(z) \prec p(z) \quad(z \in \mathbb{U})
$$

Furthermore, if

$$
\varphi\left(q(z), z p^{\prime}(z)\right)=h(z)
$$

has a univalent solution $q \in Q$, then $q$ is the best subordinat.

Lemma 1.8 (Pommerenke [16]). The function

$$
L(z, t)=a_{1}(t) z+\cdots,
$$

with

$$
a_{1}(t) \neq 0, \quad \lim _{t \rightarrow \infty}\left|a_{1}(t)\right|=\infty,
$$

is a subordination chain if and only if

$$
\mathfrak{R e}\left[\frac{z \partial L(z, t) / \partial z}{\partial L(z, t) / \partial t}\right]>0 \quad(z \in \mathbb{U} ; 0 \leqq t<\infty) .
$$

\section{Main Results}

Our first subordination is contained in Theorem 2.1. To short the formulas in this section, let us denote

$$
\partial_{\beta, \gamma}^{h}(f):=h^{\prime}(z)\left[\frac{h(z)}{z}\right]^{\gamma-1}\left[\frac{f(z)}{z}\right]^{\beta} .
$$

Theorem 2.1. Let $f, g \in \mathcal{A}_{\beta, \gamma}^{h}$. Suppose that

$$
\mathfrak{R e}\left[1+\frac{z\left[\partial_{\beta, \gamma}^{h}(g)\right]^{\prime \prime}}{\left[\partial_{\beta, \gamma}^{h}(g)\right]^{\prime}}\right]>-\delta \quad(z \in \mathbb{U}),
$$


where

$$
\delta=\frac{1+|\gamma+\beta|^{2}-\left|1-(\gamma+\beta)^{2}\right|}{4 \mathfrak{R e}(\gamma+\beta)} \quad(\mathfrak{R e}(\gamma+\beta)>0) .
$$

Then the following subordination relation:

$$
\partial_{\beta, \gamma}^{h}(f) \prec \partial_{\beta, \gamma}^{h}(g) \quad(z \in \mathbb{U})
$$

implies that

$$
\left[\frac{I_{\beta, \gamma}^{h}(f)(z)}{z}\right]^{\beta} \prec\left[\frac{I_{\beta, \gamma}^{h}(g)(z)}{z}\right]^{\beta} \quad(z \in \mathbb{U}),
$$

where $I_{\beta, \gamma}^{h}$ is the integral operator defined by (1.9). Moreover, the function $\left[I_{\beta, \gamma}^{h}(g)(z) / z\right]^{\beta}$ is the best dominant.

Proof. Let us define the functions $F$ and $G$ by

$$
F(z)=\left[\frac{I_{\beta, \gamma}^{h}(f)(z)}{z}\right]^{\beta}, \quad G(z)=\left[\frac{I_{\beta, \gamma}^{h}(g)(z)}{z}\right]^{\beta}
$$

respectively. Then

$$
G^{\prime}(z) \neq 0 \quad(|z|<1)
$$

We first show that, if the function $q$ is defined by

$$
q(z)=1+z \frac{G^{\prime \prime}(z)}{G^{\prime}(z)} \quad(z \in \mathbb{U})
$$

then

$$
\mathfrak{R e}\{q(z)\}>0 \quad(z \in \mathbb{U}) .
$$

In terms of the function $\partial_{\beta, \gamma}^{h}(g)$, the definition (1.9) readily yields

$$
\beta\left[\frac{z\left(I_{\beta, \gamma}^{h}(g)(z)\right)^{\prime}}{I_{\beta, \gamma}^{h}(g)(z)}\right]=-\gamma+(\gamma+\beta) \frac{\partial_{\beta, \gamma}^{h}(g)}{G(z)} .
$$


We also have

$$
\beta\left[\frac{z\left(I_{\beta, \gamma}^{h}(g)(z)\right)^{\prime}}{I_{\beta, \gamma}^{h}(g)(z)}\right]=\beta+\frac{z G^{\prime}(z)}{G(z)}
$$

By a simple calculation in conjunction with (2.10) and (2.11), we obtain the following relationship:

$$
1+\frac{z\left[\partial_{\beta, \gamma}^{h}(g)\right]^{\prime \prime}}{\left[\partial_{\beta, \gamma}^{h}(g)\right]^{\prime}}=q(z)+\frac{z q^{\prime}(z)}{q(z)+\gamma+\beta}=h(z)
$$

We also see from (2.2) that

$$
\mathfrak{R e}[h(z)+\gamma+\beta]>0 \quad(z \in \mathbb{U})
$$

and, by using Lemma 1.5, we conclude that the differential equation (2.12) has a solution $q \in \mathscr{\ell}(U)$ with

$$
q(0)=h(0)=1
$$

Let us put

$$
H(u, v)=u+\frac{v}{u+\gamma+\beta}+\delta
$$

where $\delta$ is given by (2.3). From (2.2), (2.12), and (2.15), we obtain

$$
\mathfrak{R e}\left[H\left(q(z), z q^{\prime}(z)\right)\right]>0 \quad(z \in \mathbb{U}) .
$$

Now we proceed to show that

$$
\mathfrak{R e}[H(i s, t)] \leq 0 \quad\left(s \in \mathbb{R} ; t \leq-\frac{1}{2}\left(1+s^{2}\right)\right) .
$$

Indeed, from (2.15), we have

$$
\mathfrak{R e}[H(i s, t)]=\mathfrak{R e}\left[i s+\frac{t}{i s+\gamma+\beta}+\delta\right]=\frac{t \mathfrak{R e}[\gamma+\beta]}{|\gamma+\beta+i s|^{2}}+\delta \leq-\frac{E_{\delta}(s)}{2|\gamma+\beta+i s|^{2}},
$$

where

$$
E_{\delta}(s)=[\mathfrak{R e}(\gamma+\beta)-2 \delta] s^{2}-4 \delta \mathfrak{I m}(\gamma+\beta) s-2 \delta|\gamma+\beta|^{2}+\mathfrak{R e}(\gamma+\beta)
$$


For $\delta$ given by (2.3), we note that the coefficient of $s^{2}$ is in the quadratic expression for $E_{\delta}(s)$ defined by (2.19) is greater than or equal to zero. Moreover, the discriminant $\Delta$ of $E_{\delta}(s)$ in (2.19) is represented by

$$
\frac{1}{4} \Delta=-4 \delta^{2}[\mathfrak{R e}(\gamma+\beta)]^{2}+2 \delta\left(1+|\gamma+\beta|^{2}\right) \mathfrak{R e}(\gamma+\beta)-[\mathfrak{R e}(\gamma+\beta)]^{2}
$$

which, for the assumed value of $\delta$ given by (2.3), yields

$$
\Delta=0,
$$

and so the quadratic expression for $s$ in $E_{\delta}(s)$ given by (2.19) is a perfect square. We thus see from (2.18) that

$$
\mathfrak{R e}[H(i s, t)] \leqq 0\left(s \in \mathbb{R} ; t \leqq-\frac{1}{2}\left(1+s^{2}\right)\right)
$$

Hence, by using Lemma 1.4, we conclude that

$$
\mathfrak{R e}[q(z)]>0 \quad(z \in \mathbb{U}),
$$

that is, the function $G$ defined by (2.6) is convex in $\mathbb{U}$.

Next, we prove that the subordination condition (2.4) implies that

$$
F(z) \prec G(z) \quad(z \in \mathbb{U})
$$

for the functions $F$ and $G$ defined by (2.6). For this purpose, we consider the function $L(z, t)$ given by

$$
L(z, t):=G(z)+\frac{1+t}{\gamma+\beta} z G^{\prime}(z) \quad(z \in \mathbb{U} ; 0 \leqq t<\infty)
$$

Since $G$ is convex in $\mathbb{U}$ and $\mathfrak{R e}(\gamma+\beta)>0$, we obtain

$$
\begin{gathered}
\left.\frac{\partial L(z, t)}{\partial z}\right|_{z=0}=G^{\prime}(0)\left(1+\frac{1+t}{\gamma+\beta}\right) \neq 0 \quad(z \in \mathbb{U} ; 0 \leqq t<\infty), \\
\mathfrak{R e}\left[\frac{z \partial L(z, t) / \partial z}{\partial L(z, t) / \partial t}\right]=\mathfrak{R e}\left\{\gamma+\beta+(1+t)\left(1+\frac{z G^{\prime \prime}(z)}{G^{\prime}(z)}\right)\right\}>0 \quad(z \in \mathbb{U}) .
\end{gathered}
$$

Therefore, by virtue of Lemma 1.8, $L(z, t)$ is a subordination chain. We observe from the definition of a subordination chain that

$$
\begin{gathered}
\partial_{\beta, \gamma}^{h}(g)(z)=G(z)+\frac{z G^{\prime}(z)}{\gamma+\beta}=L(z, 0), \\
L(z, 0) \prec L(z, t) \quad(z \in \mathbb{U} ; 0 \leqq t<\infty) .
\end{gathered}
$$


This implies that

$$
L(\zeta, t) \notin L(\mathbb{U}, 0)=\partial_{\beta, \gamma}^{h}(g)(\mathbb{U}) \quad(\zeta \in \partial \mathbb{U} ; 0 \leqq t<\infty) .
$$

Now suppose that $F$ is not subordinate to $G$. Then, by Lemma 1.6, there exist points $z \in \mathbb{U}$ and $\zeta \in \partial \mathbb{U}$ such that

$$
F\left(z_{0}\right)=G\left(\zeta_{0}\right), \quad z_{0} F^{\prime}\left(z_{0}\right)=(1+t) \zeta_{0} G^{\prime}\left(\zeta_{0}\right) \quad(0 \leqq t<\infty) .
$$

Hence, we have

$$
\begin{aligned}
L\left(\zeta_{0}, t\right) & =G\left(\zeta_{0}\right)+\left(\frac{1+t}{\gamma+\beta}\right) \zeta_{0} G^{\prime}\left(\zeta_{0}\right)=F\left(z_{0}\right)+\frac{z_{0} F^{\prime}\left(z_{0}\right)}{\gamma+\beta} \\
& =\left(\frac{h\left(z_{0}\right)}{z_{0}}\right)^{\gamma-1} h^{\prime}\left(z_{0}\right)\left(\frac{f\left(z_{0}\right)}{z_{0}}\right)^{\beta} \in \partial_{\beta, \gamma}^{h}(g) \mathbb{U},
\end{aligned}
$$

by virtue of the subordination condition (2.4). This contradicts the above observation that

$$
L\left(\zeta_{0}, t\right) \notin \partial_{\beta, \gamma}^{h}(g)(\mathbb{U}) .
$$

Therefore, the subordination condition (2.4) must imply the subordination given by (2.24). Considering $F(z)=G(z)$, we see that the function $G(z)$ is the best dominant. This evidently completes the proof of Theorem 2.1.

Remark 2.2. We note that $\delta$ given by (2.3) in Theorem 2.1 satisfies the following inequality $0<\delta \leq 1 / 2$.

Theorem 2.3. Let $f, g \in \mathcal{A}_{\beta, \gamma}^{h}$. Suppose that

$$
\mathfrak{R e}\left[1+\frac{z\left[\partial_{\beta, \gamma}^{h}(g)\right]^{\prime \prime}}{\left[\partial_{\beta, \gamma}^{h}(g)\right]^{\prime}}\right]>-\delta \quad(z \in \mathbb{U}),
$$

where $\delta$ is given by (2.3), and that the function $\partial_{\beta, \gamma}^{h}(f)$ is univalent in $\mathbb{U}$ and such that $\left[I_{\beta, \gamma}^{h}(f)(z) / z\right]^{\beta} \in Q$, where $I_{\beta, \gamma}^{h}$ is the integral operator defined by (1.9). Then the following superordination relation:

$$
\partial_{\beta, \gamma}^{h}(g) \prec \partial_{\beta, \gamma}^{h}(f) \quad(z \in \mathbb{U})
$$

implies that

$$
\left[\frac{I_{\beta, \gamma}^{h}(g)(z)}{z}\right]^{\beta} \prec\left[\frac{I_{\beta, \gamma}^{h}(f)(z)}{z}\right]^{\beta} \quad(z \in \mathbb{U}) .
$$

Moreover, the function $\left[I_{\beta, \gamma}^{h}(g)(z) / z\right]^{\beta}$ is the best subordinat. 
Proof. The first part of the proof is similar to that of Theorem 2.1 and so we will use the same notation as in the proof of Theorem 2.1. Now let us define the functions $F$ and $G$, as before, by (2.6). We first note that, by using (2.3) and (2.11), we obtain

$$
\partial_{\beta, \gamma}^{h}(g)=G(z)+\frac{z G^{\prime}(z)}{\gamma+\beta}=: \varphi\left(G(z), z G^{\prime}(z)\right) .
$$

After a simple calculation, (2.35) yields the following relationship:

$$
1+\frac{z\left[\partial_{\beta, \gamma}^{h}(g)\right]^{\prime \prime}}{\left[\partial_{\beta, \gamma}^{h}(g)\right]^{\prime}}=q(z)+\frac{z q^{\prime}(z)}{q(z)+\gamma+\beta^{\prime}}
$$

where function $q$ is defined by (2.8). Then, by using the same method as in the proof of Theorem 2.1, we can prove that

$$
\mathfrak{R e}\{q(z)\}>0 \quad(z \in \mathbb{U}),
$$

that is, $G$ defined by (2.6) is convex (univalent) in $\mathbb{U}$.

Next, we prove that the superordination condition (2.33) implies that

$$
F(z) \prec G(z) \quad(z \in \mathbb{U})
$$

For this purpose, we consider the function $L(z, t)$ defined by

$$
L(z, t)=G(z)+\frac{t}{\gamma+\beta} z G^{\prime}(z) \quad(z \in \mathbb{U} ; 0 \leqq t<\infty) .
$$

Since $G$ is convex and $\mathfrak{R e}(\gamma+\beta)>0$, we can prove easily that $L(z, t)$ is a subordination chain as in the proof of Theorem 2.1. Therefore, according to Lemma 1.7, we conclude that the superordination condition (2.33) must imply the superordination given by (2.38). Furthermore, since the differential equation (2.35) has the univalent solution $G$, it is the best subordinat of the given differential subordination. We thus complete the proof of Theorem 2.3.

If we suitably combine Theorems 2.1 and 2.3, then we obtain the following sandwichtype theorem.

Theorem 2.4. Let $f, g_{1}, g_{2} \in \mathcal{A}_{\beta, \gamma}^{h}$. Suppose that

$$
\mathfrak{R e}\left[1+\frac{z\left[\partial_{\beta, \gamma}^{h}\left(g_{1}\right)\right]^{\prime \prime}}{\left[\partial_{\beta, \gamma}^{h}\left(g_{1}\right)\right]^{\prime}}\right]>-\delta, \quad \mathfrak{R e}\left[1+\frac{z\left[\partial_{\beta, \gamma}^{h}\left(g_{2}\right)\right]^{\prime \prime}}{\left[\partial_{\beta, \gamma}^{h}\left(g_{2}\right)\right]^{\prime}}\right]>-\delta \quad(z \in \mathbb{U}),
$$


where $\delta$ is given by (2.3), and that the function $\partial_{\beta, \gamma}^{h}(f)$ is univalent in $\mathbb{U}$ and such that $\left[I_{\beta, \gamma}^{h}(f)(z) / z\right]^{\beta} \in \mathcal{Q}$, where $I_{\beta, \gamma}^{h}$ is the integral operator defined by (1.9). Then the following subordination relation:

$$
\partial_{\beta, \gamma}^{h}\left(g_{1}\right) \prec \partial_{\beta, \gamma}^{h}(f) \prec \partial_{\beta, \gamma}^{h}\left(g_{2}\right) \quad(z \in \mathbb{U})
$$

implies that

$$
\left[\frac{I_{\beta, \gamma}^{h}\left(g_{1}\right)(z)}{z}\right]^{\beta} \prec\left[\frac{I_{\beta, \gamma}^{h}(f)(z)}{z}\right]^{\beta}<\left[\frac{I_{\beta, \gamma}^{h}\left(g_{2}\right)(z)}{z}\right]^{\beta} \quad(z \in \mathbb{U}) .
$$

Moreover, the functions $\left[I_{\beta, \gamma}^{h}\left(g_{1}\right)(z) / z\right]^{\beta}$ and $\left[I_{\beta, \gamma}^{h}\left(g_{2}\right)(z) / z\right]^{\beta}$ are the best subordinat and the best dominant, respectively.

The assumption of Theorem 2.4, that the functions $\partial_{\beta, r}^{h}(f)$ and $\left[I_{\beta, \gamma}^{h}(f)(z) / z\right]^{\beta}$ need to be univalent in $U$, may be replaced by another condition in the following result.

Corollary 2.5. Let $f, g_{1}, g_{2} \in \mathcal{A}_{\beta, \gamma}^{h}$. Suppose that the condition (2.49) is satisfied and that $f(z) / z \in$ $Q$ and

$$
\mathfrak{R e}\left[1+\frac{z\left[\partial_{\beta, \gamma}^{h}(f)\right]^{\prime \prime}}{\left[\partial_{\beta, \gamma}^{h}(f)\right]^{\prime}}\right]>\delta \quad(z \in \mathbb{U})
$$

where $\delta$ is given by (2.3). Then the following subordination relation:

$$
\partial_{\beta, \gamma}^{h}\left(g_{1}\right) \prec \partial_{\beta, \gamma}^{h}(f)<\partial_{\beta, \gamma}^{h}\left(g_{2}\right) \quad(z \in \mathbb{U})
$$

implies that

$$
\left[\frac{I_{\beta, \gamma}^{h}\left(g_{1}\right)(z)}{z}\right]^{\beta} \prec\left[\frac{I_{\beta, \gamma}^{h}(f)(z)}{z}\right]^{\beta} \prec\left[\frac{I_{\beta, \gamma}^{h}\left(g_{2}\right)(z)}{z}\right]^{\beta} \quad(z \in \mathbb{U}),
$$

where $I_{\beta, r}^{h}$ is the integral operator defined by (1.9). Moreover, the functions $\left[I_{\beta, \gamma}^{h}\left(g_{1}\right)(z) / z\right]^{\beta}$ and $\left[I_{\beta, \gamma}^{h}\left(\left(g_{2}\right)(z) / z\right)\right]^{\beta}$ are the best subordinat and the best dominant, respectively.

Proof. In order to prove Corollary 2.5, we have to show that the condition (2.43) implies the univalence of each of the functions $\partial_{\beta, \gamma}^{h}(f)$ and $F(z)=\left[I_{\beta, \gamma}^{h}(f)(z) / z\right]^{\beta}$.

Since $0<\delta \leq 1 / 2$, just as in Remark 2.2, the condition (2.43) means that $\psi$ is a close-toconvex function in $\mathbb{U}$ (see [17]), and hence $\partial_{\beta, \gamma}^{h}(f)$ is univalent in $\mathbb{U}$. Furthermore, by using the same techniques as in the proof of Theorem 2.4, we can prove the convexity (univalence) 
of $F$, and so the details are being omitted here. Thus, by applying Theorem 2.4, we readily obtain Corollary 2.5.

By setting $\gamma+\beta=2$ in Theorem 2.4, so that $\delta=1 / 4$, we deduce the following consequence of Theorem 2.4.

Corollary 2.6. Let $f, g_{1}, g_{2} \in \mathcal{A}_{\beta, 2-\beta}^{h}$. Suppose that

$$
\mathfrak{R e}\left[1+\frac{z\left[\partial_{\beta, \gamma}^{h}\left(g_{1}\right)\right]^{\prime \prime}}{\left[\partial_{\beta, \gamma}^{h}\left(g_{1}\right)\right]^{\prime}}\right]>-\frac{1}{4}, \quad \mathfrak{R e}\left[1+\frac{z\left[\partial_{\beta, \gamma}^{h}\left(g_{2}\right)\right]^{\prime \prime}}{\left[\partial_{\beta, \gamma}^{h}\left(g_{2}\right)\right]^{\prime}}\right]>-\frac{1}{4} \quad(z \in \mathbb{U}),
$$

and that the function $\partial_{\beta, \gamma}^{h}(f)$ is univalent in $\mathbb{U}$ and $\left[I_{\beta, 2-\beta}^{h}(f)(z) / z\right]^{\beta} \in Q$, where $I_{\beta, 2-\beta}^{h}$ is the integral operator defined by (1.9) with $\gamma=\beta+2$. Then the following subordination relation:

$$
\partial_{\beta, \gamma}^{h}\left(g_{1}\right) \prec \partial_{\beta, \gamma}^{h}(f) \prec \partial_{\beta, \gamma}^{h}\left(g_{2}\right) \quad(z \in \mathbb{U})
$$

implies that

$$
\left[\frac{I_{\beta, 2-\beta}^{h}\left(g_{1}\right)(z)}{z}\right]^{\beta} \prec\left[\frac{I_{\beta, 2-\beta}^{h}(f)(z)}{z}\right]^{\beta} \prec\left[\frac{I_{\beta, 2-\beta}^{h}\left(g_{2}\right)(z)}{z}\right]^{\beta} \quad(z \in \mathbb{U}) .
$$

Moreover, the functions $\left[I_{\beta, 2-\beta}^{h}\left(g_{1}\right)(z) / z\right]^{\beta}$ and $\left[I_{\beta, 2-\beta}^{h}\left(g_{2}\right)(z) / z\right]^{\beta}$ are the best subordinat and the best dominant, respectively.

If we take $\gamma+\beta=1+i$ in Theorem 2.4, then we are easily led to the following result.

Corollary 2.7. Let $f, g_{1}, g_{2} \in \mathcal{A}_{\beta, 1+i-\beta}^{h}$. Suppose that

$$
\mathfrak{R e}\left[1+\frac{z\left[\partial_{\beta, \gamma}^{h}\left(g_{1}\right)\right]^{\prime \prime}}{\left[\partial_{\beta, \gamma}^{h}\left(g_{1}\right)\right]^{\prime}}\right]>\frac{\sqrt{5}-3}{4}, \quad \mathfrak{R e}\left[1+\frac{z\left[\partial_{\beta, \gamma}^{h}\left(g_{2}\right)\right]^{\prime \prime}}{\left[\partial_{\beta, \gamma}^{h}\left(g_{2}\right)\right]^{\prime}}\right]>\frac{\sqrt{5}-3}{4} \quad(z \in \mathbb{U}),
$$

and that the function $\partial_{\beta, \gamma}^{h}(f)$ is univalent in $\mathbb{U}$ and $\left[I_{\beta, 1+i-\beta}^{h}(f)(z) / z\right]^{\beta} \in \mathcal{Q}$, where $I_{\beta, 1+i-\beta}^{h}$ is the integral operator defined by (1.9) with $\gamma=\beta+2$. Then the following subordination relation:

$$
\partial_{\beta, \gamma}^{h}\left(g_{1}\right) \prec \partial_{\beta, \gamma}^{h}(f) \prec \partial_{\beta, \gamma}^{h}\left(g_{2}\right) \quad(z \in \mathbb{U})
$$

implies that

$$
\left[\frac{I_{\beta, 1+i-\beta}^{h}\left(g_{1}\right)(z)}{z}\right]^{\beta} \prec\left[\frac{I_{\beta, 1+i-\beta}^{h}(f)(z)}{z}\right]^{\beta} \prec\left[\frac{I_{\beta, 1+i-\beta}^{h}\left(g_{2}\right)(z)}{z}\right]^{\beta} \quad(z \in \mathbb{U}) .
$$


Moreover, the functions $\left[I_{\beta, 1+i-\beta}^{h}\left(g_{1}\right)(z) / z\right]^{\beta}$ and $\left[I_{\beta, 1+i-\beta}^{h}\left(g_{2}\right)(z) / z\right]^{\beta}$ are the best subordinat, respectively.

\section{Application to the Gauss Hypergeometric Function}

We begin by recalling that the Gauss hypergeometric function ${ }_{2} F_{1}(a, b ; c ; z)$ is defined by (see, for details, [18] and [19, Chapter 14])

$$
{ }_{2} F_{1}(a, b ; c ; z)=\sum_{n=0}^{\infty} \frac{(a)_{n}(b)_{n}}{(c)_{n}} \frac{z^{n}}{n !} \quad\left(z \in \mathbb{U} ; a, b \in \mathbb{C} ; c \in \mathbb{C} \backslash \mathbb{Z}_{0}^{-} ; \mathbb{Z}_{0}^{-}=\{0,-1,-2, \ldots\}\right),
$$

where $(\lambda)_{v}$ denotes the Pochhammer symbol (or the shifted factorial) defined (for $\lambda, v \in \mathbb{C}$ and in terms of the Gamma function) by

$$
(\lambda)_{v}=\frac{\Gamma(\lambda+v)}{\Gamma(\lambda)}= \begin{cases}1 & (v=0 ; \lambda \in \mathbb{C} \backslash\{0\}) \\ \lambda(\lambda+1) \cdots(\lambda+v-1) & (v=n \in \mathbb{N} ; \lambda \in \mathbb{C})\end{cases}
$$

For this useful special function, the following Eurlerian integral representation is fairly well known [19, page. 293]:

$$
\begin{aligned}
{ }_{2} F_{1}(a, b ; c ; z) & =\frac{\Gamma(c)}{\Gamma(a) \Gamma(c-a)} \int_{0}^{1} t^{a-1}(1-t)^{c-a-1}(1-z t)^{-b} d t \\
(\mathfrak{R e}(c) & >\mathfrak{R e}(a)>0 ;[\arg (1-z)] \leq \pi-\varepsilon ; 0<\varepsilon<\pi) .
\end{aligned}
$$

In view of (3.3), we set

$$
g(z)=\frac{z}{(1-z)^{k}} \quad(k>0)
$$

$\gamma=0$ and $h(z)=z e^{-z}$, so that the definition (1.9) yields

$$
\begin{aligned}
I_{\beta} & =\left(\beta \int_{0}^{z} t^{\beta-1}(1-t)^{1-k \beta} d t\right)^{1 / \beta}=z\left(\beta \int_{0}^{1} u^{\beta-1}(1-z u)^{1-k \beta} d u\right)^{1 / \beta} \\
& =z\left[{ }_{2} F_{1}(\beta, k \beta-1, \beta+1 ; z)\right]^{1 / \beta} .
\end{aligned}
$$

Moreover, we note that

$$
\frac{g(z)}{z}=\frac{1}{(1-z)^{k}} \neq 0 \quad(z \in \mathbb{U})
$$

and for $\beta>0$ the condition (2.2) becomes $k \beta \leq 2(\delta+1)$. Therefore, by applying Theorem 2.1 with $g(z)=z /(1-z)^{k},(k>0)$, we obtain the following result. 
Theorem 3.1. Let $f \in \mathcal{A}_{\beta, \gamma}^{h}$ with $\gamma=0, \beta>0, h(z)=z e^{-z}$. Suppose that

$$
k \beta \leq 2(\delta+1) \quad(k>0),
$$

where

$$
\delta=\frac{1+\beta^{2}-\left|1-\beta^{2}\right|}{4 \beta}
$$

Then the following subordination relation:

$$
(1-z)\left[\frac{f(z)}{z}\right]^{\beta} \prec \frac{1}{(1-z)^{k \beta-1}} \quad(z \in \mathbb{U})
$$

implies that

$$
\left[\frac{I_{\beta}(f)(z)}{z}\right]^{\beta} \prec{ }_{2} F_{1}(\beta, k \beta-1 ; \beta+1 ; z) \quad(z \in \mathbb{U})
$$

where $I_{\beta}$ is the integral operator defined by (1.9). Moreover, the function ${ }_{2} F_{1}(\beta, k \beta-1 ; \beta+1 ; z)$ is the best dominant.

For $\beta=1$ we get $\delta=1 / 2$ and Theorem 3.1 becomes the following Corollary.

Corollary 3.2. Let $f \in \mathcal{A}_{1,0}^{h}$ and $0<k \leq 3$. Then the following subordination relation:

$$
(1-z)\left(\frac{f(z)}{z}\right) \prec \frac{1}{(1-z)^{k-1}} \quad(z \in \mathbb{U})
$$

implies that

$$
\left(\frac{I_{1}(f)(z)}{z}\right) \prec{ }_{2} F_{1}(1, k-1 ; 2, z) \quad(z \in \mathbb{U}),
$$

where the integral operator $I_{1}$ is defined by (1.9).

We state the following result as the dual result of Theorem 3.1, which can be obtained by similarly applying Theorem 2.3 .

Theorem 3.3. Under the assumption of Theorem 3.1, suppose also that the function $(1-z)[f(z) / z]^{\beta}$ is univalent in $\mathbb{U}$ and that $\left[I_{\beta}(f)(z) / z\right]^{\beta} \in Q$, where $I_{\beta}$ is the integral operator defined by (1.9). Then the following superordination relation:

$$
\frac{1}{(1-z)^{k \beta-1}} \prec(1-z)\left[\frac{f(z)}{z}\right]^{\beta} \quad(z \in \mathbb{U})
$$


implies that

$$
{ }_{2} F_{1}(\beta, k \beta-1 ; \beta+1 ; z) \prec\left[\frac{I_{\beta}(f)(z)}{z}\right]^{\beta} \quad(z \in \mathbb{U}) .
$$

Moreover, the function ${ }_{2} F_{1}(\beta, k \beta-1 ; \beta-1 ; z)$ is the best subordinat.

If we set in (1.9) $g(z)=z /(1-z)^{k}(k>0), \gamma=0$ and $h(z)=z /(1-z)$, then we get

$$
\begin{aligned}
I_{\beta}=\left(\beta \int_{0}^{z} t^{\beta-1}(1-t)^{-1-k \beta} d t\right)^{1 / \beta} & =z\left(\beta \int_{0}^{1} u^{\beta-1}(1-z u)^{-1-k \beta} d u\right)^{1 / \beta} \\
& =z\left[{ }_{2} F_{1}(\beta, k \beta+1 ; \beta+1 ; z)\right]^{1 / \beta} .
\end{aligned}
$$

Therefore by applying Theorem 2.1, we obtain the following result.

Theorem 3.4. Let $f \in \mathcal{A}_{\beta, \gamma}^{h}$ with $\gamma=0, \beta>0, h(z)=z /(1-z)$. Suppose that $k>0$ and

$$
k \beta \leq 2 \delta
$$

where

$$
\delta=\frac{1+\beta^{2}-\left|1-\beta^{2}\right|}{4 \beta} .
$$

Then the following subordination relation:

$$
\frac{1}{1-z}\left[\frac{f(z)}{z}\right]^{\beta} \prec \frac{1}{(1-z)^{k \beta+1}} \quad(z \in \mathbb{U})
$$

implies that

$$
\left[\frac{I_{\beta}(f)(z)}{z}\right]^{\beta} \prec{ }_{2} F_{1}(\beta, k \beta+1 ; \beta+1 ; z) \quad(z \in \mathbb{U})
$$

where $I_{\beta}$ is the integral operator defined by (1.9). Moreover, the function ${ }_{2} F_{1}(\beta, k \beta+1 ; \beta+1 ; z)$ is the best dominant.

By taking $\beta=1$ in Theorem 3.1, we are led to Corollary 3.5.

Corollary 3.5. Let $f \in \mathcal{A}_{\beta, \gamma}^{h}$ with $\gamma=0, \beta=1, h(z)=z /(1-z)$. Then the following subordination relation:

$$
\frac{1}{z(1-z)} f(z) \prec \frac{1}{(1-z)^{2}} \quad(z \in \mathbb{U})
$$


implies that

$$
\left(\frac{I_{1}(f)(z)}{z}\right) \prec{ }_{2} F_{1}(1,2 ; 2 ; z) \quad(z \in \mathbb{U})
$$

where the integral operator $I_{1}$ is defined by (1.9).

We state the following result as the dual result of Theorem 3.4, which can be obtained by similarly applying Theorem 2.3.

Theorem 3.6. Under the assumption of Theorem 3.4, suppose also that the function $(1 /(1-$ $z))[f(z) / z]^{\beta}$ is univalent in $\mathbb{U}$ and that $\left[I_{\beta}(f)(z) / z\right]^{\beta} \in \mathcal{Q}$, where $I_{\beta}$ is the integral defined by (1.9) if we take $\gamma=0$. Then the following superordination relation:

$$
\frac{1}{(1-z)^{k \beta+1}} \prec \frac{1}{1-z}\left[\frac{f(z)}{z}\right]^{\beta} \quad(z \in \mathbb{U})
$$

implies that

$$
{ }_{2} F_{1}(\beta, k \beta+1 ; \beta+1 ; z) \prec\left[\frac{I_{\beta}(f)(z)}{z}\right]^{\beta} \quad(z \in \mathbb{U}) .
$$

Moreover, the function ${ }_{2} F_{1}(\beta, k \beta+1, \beta+1 ; z)$ is the best subordinat.

\section{Acknowledgment}

This paper was supported by the Science Research Program in Science College in Dammam University, Saudi Arabia.

\section{References}

[1] S. S. Miller and P. T. Mocanu, Differential Subordinations, Theory and Applications, vol. 225 of Monographs and Textbooks in Pure and Applied Mathematics, Marcel Dekker, New York, NY, USA, 2000.

[2] S. S. Miller and P. T. Mocanu, "Subordinants of differential superordinations," Complex Variables: Theory and Application, vol. 48, no. 10, pp. 815-826, 2003.

[3] J. W. Alexander, "Functions which map the interior of the unit circle upon simple regions," Annals of Mathematics Second Series, vol. 17, no. 1, pp. 12-22, 1915.

[4] S. D. Bernardi, "Convex and starlike univalent functions," Transactions of the American Mathematical Society, vol. 135, pp. 429-446, 1969.

[5] B. C. Carlson and D. B. Shaffer, "Starlike and prestarlike hypergeometric functions," SIAM Journal on Mathematical Analysis, vol. 15, no. 4, pp. 737-745, 1984.

[6] R. J. Libera, "Some classes of regular univalent functions," Proceedings of the American Mathematical Society, vol. 16, pp. 755-758, 1965.

[7] T. Bulboacă, "On a special integral subordination," Indian Journal of Pure and Applied Mathematics, vol. 28, no. 3, pp. 361-369, 1997.

[8] T. Bulboacă, "Integral operators that preserve the subordination," Bulletin of the Korean Mathematical Society, vol. 34, no. 4, pp. 627-636, 1997. 
[9] T. Bulboacă, "On a class of integral operators that preserve the subordination," Pure Mathematics and Applications, vol. 13, no. 1-2, pp. 87-96, 2002, Algebraic systems (Felix-Oradea, 2001).

[10] T. Bulboacă, "A class of superordination-preserving integral operators," Koninklijke Nederlandse Akademie van Wetenschappen. Indagationes Mathematicae New Series, vol. 13, no. 3, pp. 301-311, 2002.

[11] T. Bulboacă, "A class of double subordination-preserving integral operators," Pure Mathematics and Applications, vol. 15, no. 2-3, pp. 87-106, 2004.

[12] T. Bulboacă, "Sandwich-type theorems for a class of integral operators," Bulletin of the Belgian Mathematical Society Simon Stevin, vol. 13, no. 3, pp. 537-550, 2006.

[13] N. E. Cho and S. Owa, "Double subordination-preserving properties for certain integral operators," Journal of Inequalities and Applications, vol. 2007, Article ID 83073, 10 pages, 2007.

[14] S. S. Miller and P. T. Mocanu, "Differential subordinations and univalent functions," The Michigan Mathematical Journal, vol. 28, no. 2, pp. 157-172, 1981.

[15] S. S. Miller and P. T. Mocanu, "Univalent solutions of Briot-Bouquet differential equations," Journal of Differential Equations, vol. 56, no. 3, pp. 297-309, 1985.

[16] C. Pommerenke, Univalent Functions, Vandenhoeck \& Ruprecht, Göttingen, Germany, 1975.

[17] W. Kaplan, "Close-to-convex schlicht functions," The Michigan Mathematical Journal, vol. 1, no. 2, pp. $169-185,1952$.

[18] S. Owa and H. M. Srivastava, "Univalent and starlike generalized hypergeometric functions," Canadian Journal of Mathematics, vol. 39, no. 5, pp. 1057-1077, 1987.

[19] E. T. Whittaker and G. N. Watson, A Course of Modern Analysis, an Introduction to the General Theory of Infinite Processes and of Analytic Functions, with an Account of the Principal Transcendental Functions, Cambridge University Press, Cambridge, UK, 4th edition, 1927. 


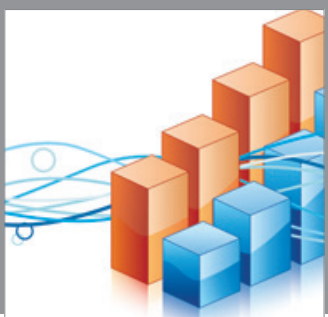

Advances in

Operations Research

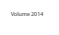

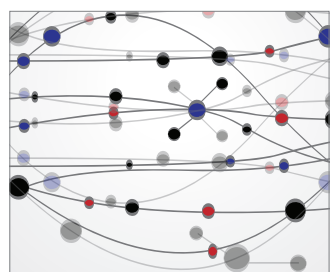

\section{The Scientific} World Journal
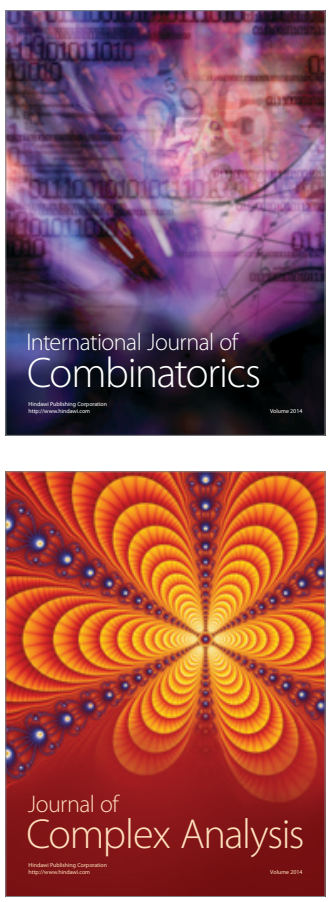

International Journal of

Mathematics and

Mathematical

Sciences
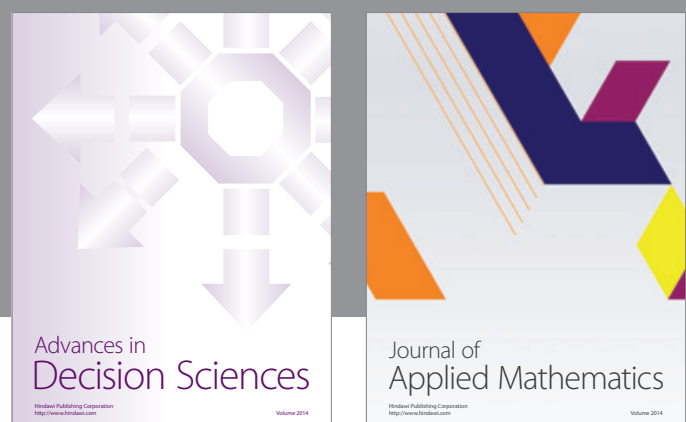

Journal of

Applied Mathematics
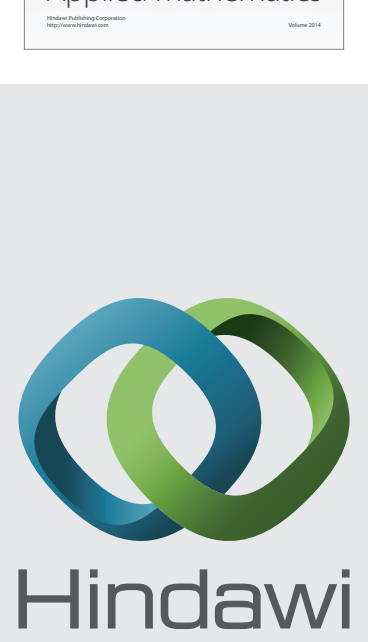

Submit your manuscripts at http://www.hindawi.com
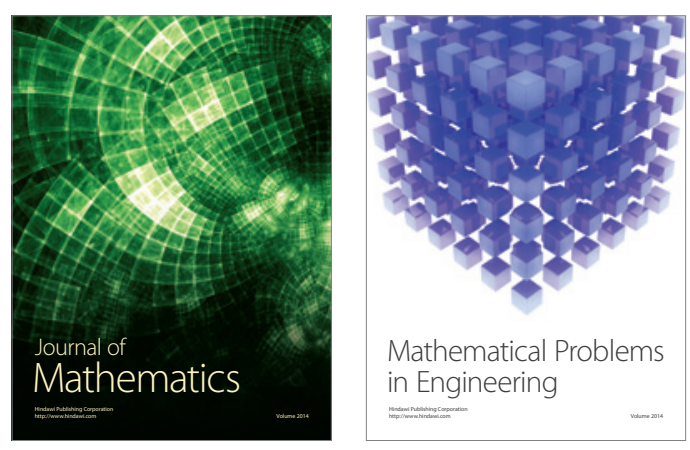

Mathematical Problems in Engineering
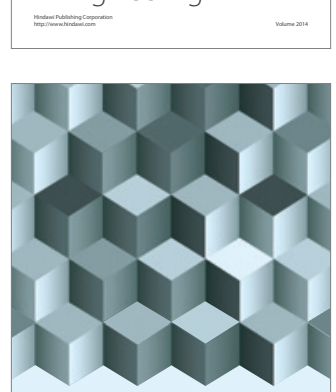

Journal of

Function Spaces
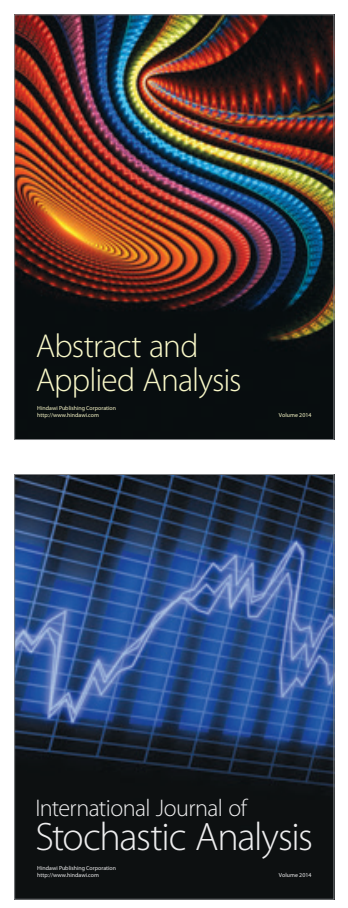

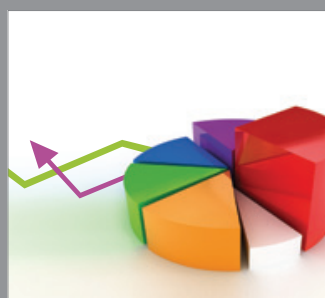

ournal of

Probability and Statistics

Promensencen
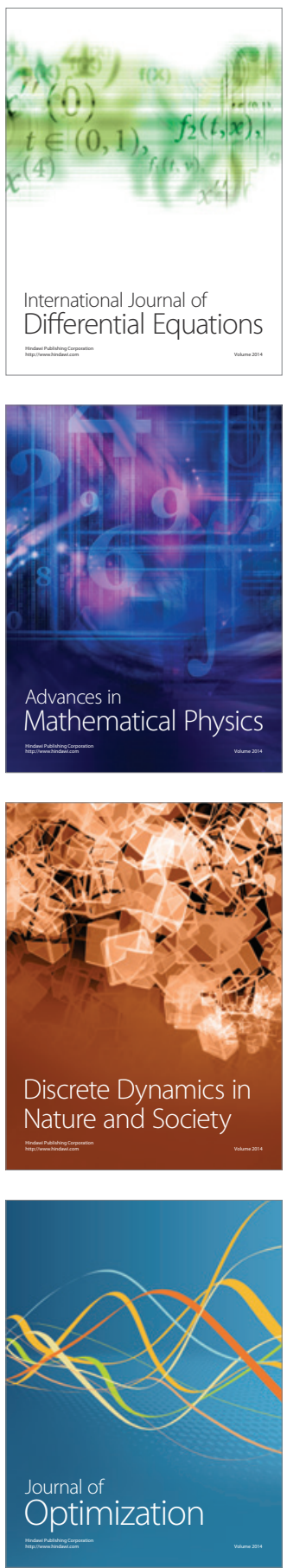